\title{
Erratum to: Adaptation to Coastal Storms in Atlantic Canada
}

Liette Vasseur, Mary J. Thornbush and Steve Plante

\section{Erratum to:}

L. Vasseur et al., Adaptation to Coastal Storms in Atlantic Canada, SpringerBriefs in Geography, DOI 10.1007/978-3-319-63492-0

At the end of the Frontmatter of the original book version, an Acknowledgement has been added as a belated correction.

The currently available book version has been updated with this change. 